\title{
Cosmology
}

\section{Ever more distant quasars?}

\section{Peter Shaver}

THE distances to far-off objects in the expanding Universe are measured by their large redshifts $(z)$ - the fractional increases in the wavelength of the light they emit. There are now six quasars known with redshifts greater than 4; all have been found within the past year. Two of them, including the quasar with the highestknown redshift $(z=4.43)$, are reported on page 453 of this issue ${ }^{1}$ by Warren et al. This rapid proliferation of high-redshift quasars is largely the result of improved search techniques, whose redshift limits are already being approached. It demonstrates that a redshift cutoff has not yet been found, and suggests that the space density of quasars may not decrease substantially towards these higher redshifts.

The space density of quasars above a given luminosity increases rapidly up to $z \simeq 2$. The first quasar with $z \geqslant 2$ was only the ninth quasar known. Although the number of known quasars increased rapidly, the highest redshifts for several years were still not much above 2 , giving rise to the notion of a redshift cutoff at $z \simeq 2$ and speculation as to the possible cause. The quasar OQ172, found in 1973, has a redshift of 3.53 , and held the record for almost a decade, during which time more than 1,500 quasars were found. This record was finally broken in 1982 by PKS 2000-330, which has a redshift of 3.78. Such high redshifts were, however, apparently rare, and it became widely believed that the space density of quasars peaked at $z \simeq 2$, the 'quasar epoch' and fell off at higher redshift (light from distant objects must have been emitted a correspondingly long time ago).

\section{Optical searches}

All these redshift records were set by radio-loud quasars discovered through their radio emission; but most quasars are radio-quiet. Optical techniques are extremely efficient for finding quasars, but, until recently, have been biased against finding quasars with $z>3$. One such technique is based on broadband colours: quasars have relatively blue continua, so 'UVX' quasar candidates are selected on the basis of an ultraviolet excess. The other technique is based on slitless spectroscopy: quasars have prominent emission lines, and 'objective prism' or 'grism' candidates are identified on the basis of both blue continua and characteristic emission features in low dispersion spectra. Both techniques are readily applied to photographic plates covering large areas, and digital scanning of these plates with computer analysis increases the efficiency of these searches.

At higher redshifts the most prominent emission lines are shifted far to the red, and the ultraviolet excess disappears because of strong absorption by intervening hydrogen. Several recent quasar searches have therefore been made using both broadband colours and slitless spectroscopy at longer wavelengths, some using red-sensitive photographic plates ${ }^{1-3}$ and others scanning charge-coupled devices $^{4}$; these make possible the detection of quasars out to redshifts of 4.5-5.0.

The promise of these new techniques has been realized with the recent burst of high-redshift discoveries (see table), and

Known quasars with $z>4$;

$m_{\mathrm{R}}$, magnitude (brightness) in the red.

\begin{tabular}{lcccc}
\hline Quasar & $z$ & $m_{\mathrm{R}}$ & Method & Ref. \\
$0046-293$ & 4.01 & 19 & colour & 2 \\
$0910+564$ & 4.04 & 21 & spectroscopy & 4 \\
$0101-304$ & 4.07 & 19.5 & colour & 1 \\
$0000-263$ & 4.11 & $\simeq 18$ & spectroscopy & $*$ \\
$2203+292$ & 4.40 & 21 & serendipity & $\dagger$ \\
$0051-279$ & 4.43 & 20 & colour & 1 \\
\hline
\end{tabular}

* Hazard, C. et al., in preparation.

† McCarthy, P. et al., in preparation.

much of the redshift range over which they are sensitive has already been covered. These high-redshift quasars are relatively luminous, although not the most luminous known. They also appear to be surprisingly abundant, judging from the fact that three of them were found on just one $6^{\circ} \times 6^{\circ}$ plate, and the amazing serendipitous discovery by $\mathrm{P}$. McCarthy and $\mathrm{M}$. Dickinson (personal communication) of another whose spectrum was obtained by chance during a long-slit observation of a $z=0.8$ radio galaxy. This is consistent with some recent analyses according to which the space density of bright quasars may still be increasing at high redshifts ${ }^{3,5}$.

The observed space density of highredshift quasars has a direct bearing on such issues as galaxy formation, obscuration by intervening matter, and gravitational lensing - in addition to the evolution of quasars themselves. The very existence of quasars of high redshift is awkward for some theories which predict relatively late formation of galaxies, such as those based on cold, dark matter. The spectra of the high-redshift quasars are not markedly different from those of others at lower redshifts, and the presence of similar heavy-element absorption lines due to intervening high-redshift matter indicates enrichment by still earlier generations of stars. The first galaxies apparently formed well before the time corresponding to $z \simeq 4$ when the Universe was less than 10-20 per cent of its present age.

Studies of faint galaxies and absorption systems in quasar spectra indicate that galaxies up to $z \simeq 4-5$ may cover almost the whole sky. If these contain dust they could obscure distant quasars ${ }^{6}$, and this could explain the fact that the space density of known quasars is insufficient to produce the high degree of ionization of the intergalactic medium at high redshifts. The known high-redshift quasars, however, do not appear to be significantly reddened by absorption ${ }^{7}$ (with the possible exception of Q0051-279 itself), and any actual redshift cutoff due to obscuration must evidently be in excess of $z=4-5$.

\section{Gravitational lensing}

The opposite effect, an apparent increase in the space density of luminous quasars, could conceivably be produced through magnification by gravitational lensing. Progress in understanding these issues will require the laborious assembly of statistically complete samples of quasars at these high redshifts, now possible with the new techniques.

What are the prospects for pursuing the quasar search to still higher redshifts? Further adaptation of the above methods, augmented by near-infrared photometry and narrowband imaging, could extend the accessible redshift range to $z \simeq 6$. At $z=7$, hydrogen Lyman- $\alpha$ lines would be redshifted to almost $1 \mu \mathrm{m}$, and the entire optical band would probably be heavily obscured by absorption by hydrogen along the line of sight. Spectroscopic confirmation, as a result, would have to be made in the near infrared. Searches could in principle also be made in the infrared, although the relevant technology is still in its infancy.

Alternatively, one could concentrate on 'blank-field' X-ray or radio sources: that is, those with no optical counterparts to very faint limits, Virtually all quasars are $\mathrm{X}$-ray sources, but those at high redshifts will be weak, and a sufficiently sensitive satellite has yet to be launched. Blankfield radio sources offer more immediate prospects, although the number of radioloud quasars at high redshifts is probably very small. The recent successes at redshifts of 4-5 will undoubtedly stimulate such efforts.

1. Warren, S.J., Hewett, P.C., Osmer, P.S. \& Irwin, M.J. Nature 330, 453-455 (1987)

2. Warren, S.J. et al. Noture 325, 131-133 (1987).

3. Hazard, C., McMahon, R.G. \& Sargent, W.L.W. Nature 322, 38-40 (1986).

4chmidt, M., Schneider, D.P. \& Gunn, J.E. Astrophys. J. 321, L7-L10 (1987)

5. Koo, D.C. \& Kron, R.G. Astrophys. J. (in the press).

6. Ostriker, J.P. \& Heisler, J. Astrophys. J. 278, 1-10 (1984).

7. Wright, E.L. \& Malkan, M.A. Bull. Am. astr. Soc. 19,699 (1987)

Peter Shaver is at the European Southern Observatory, Karl-Schwarzchild-Strasse 2, D-8046 Garching bei München, FRG. 